\title{
BMJ Open Quality Introduction of a modified obstetric early warning system (MOEWS) at an Ethiopian referral hospital: a feasibility assessment
}

\author{
Jolene Moore, ${ }^{1}$ Duncan Thomson, ${ }^{1}$ Iona Pimentil, ${ }^{1}$ Bazezew Fekad, ${ }^{2}$ \\ Wendy Graham ${ }^{1,3}$
}

To cite: Moore J, Thomson D, Pimentil I, et al. Introduction of a modified obstetric early warning system (MOEWS) at an Ethiopian referral hospital: a feasibility assessment. BMJ Open Quality 2019:8:e000503. doi:10.1136/ bmjoq-2018-000503

Received 10 August 2018 Revised 5 February 2019 Accepted 8 March 2019

\section{Check for updates}

(C) Author(s) (or their employer(s)) 2019. Re-use permitted under CC BY-NC. No commercial re-use. See rights and permissions. Published by BMJ.

${ }^{1}$ College of Life Sciences and Medicine, University of Aberdeen, Aberdeen, UK ${ }^{2}$ Department of Obstetrics and Gynaecology, Felege Hiwot Regional Referral Hospital, Bahir Dar, Ethiopia

${ }^{3}$ Faculty of Epidemiology and Population Health, London School of Hygiene \& Tropical Medicine, London, UK

Correspondence to Dr Jolene Moore; jolenemoore@abdn.ac.uk

\section{ABSTRACT}

Early warning scores are points-based or colour-coded systems used to detect changes in physiological parameters and prompt earlier recognition and management of deteriorating patients. Vital signs recorded within a coloured zone corresponding to degree of derangement ('trigger') should prompt an action. The report of the UK Confidential Enquiry into Maternal and Child Health recommends the use of modified versions in the obstetric population. Currently, there is limited research into the effects of early warning scores in lowresource settings where maternal mortality remains high, and there is a need for low-cost, simple methods to reduce this. A modified obstetric early warning system (MOEWS) was introduced for parturients who had undergone surgical intervention at Felege Hiwot Referral Hospital, a tertiary centre in Bahir Dar, Ethiopia. A guideline was developed to accompany the MOEWS, together with training of healthcare workers. Prior to introduction, the quality of postoperative monitoring was assessed through retrospective case note review. This was reassessed at 8 months and 11 months postimplementation, with assessment of response to 'triggers'. A questionnaire and qualitative interviews were undertaken to establish views of healthcare workers on its acceptability and usability. Recording of postoperative vital signs improved with the implementation of the MOEWS and was sustained at both monitoring periods. The number of patients with vital signs within the coloured zones ('trigger') was reduced, although documented action to these remained low. Staff were positive towards the MOEWS, its impact on patient care and felt confident using the system. The introduction of a MOEWS in an Ethiopian referral hospital in this study appeared to improve the monitoring of postoperative patients. With modifications to suit the setting and senior clinician involvement, coupled with regular training, the early warning score is a feasible and acceptable tool to cope with the unique demands faced in this low-resource setting.

\section{THE PROBLEM}

Preventing maternal mortality remains a significant challenge in many parts of the world, particularly low-income countries where resources are limited. Sadly, many of these deaths are preventable. One way to prevent maternal deaths in healthcare settings is to detect physiological parameters suggestive of a decline in condition before such changes become irreversible and fatal. Early warning or 'track and trigger' systems have been designed to prompt earlier recognition and management of deteriorating patients. There is currently very little literature considering the impact of such systems in low-resource settings.

Ethiopia has a population of almost 100 million people making it the second most populous country in Africa. ${ }^{1}$ The Maternal Mortality Ratio (MMR) in Ethiopia is among the highest in the world despite a notable reduction in recent years. There are various estimates of the national MMR for Ethiopia; the figure from the latest Ethiopian Demographic and Health Survey (2016) is 416 maternal deaths per 100000 live births for the period spanning 2009-2016. ${ }^{2}$ Felege Hiwot Referral Hospital (FHRH) is one of 82 referral hospitals across the whole of Ethiopia and serves a population of 7 million people. FHRH has a delivery rate of almost 6000 per year and a caesarean section rate of $35 \%-40 \%$, equating to between 2000 and 2400 caesarean sections per year. The facility-based MMR rate has been between 300 and 400 per 100000 for the past 3 years (FHRH Obstetric Department \& FHRH Management, personal communication, 2019).

The aim of this study was to introduce a modified obstetric early warning system (MOEWS) in this facility and assess its feasibility and potential impact on improving postoperative monitoring and earlier identification and management of deteriorating patients.

\section{BACKGROUND}

It is well known that patients usually demonstrate preceding changes in physiological 
parameters prior to significant deterioration into a critical condition. Early warning system (EWS) or 'track and trigger' systems use numerical or colour coded systems to alert staff to dangerous trends in a patient's physiological parameters and thus have the potential to identify patients at risk of further deterioration. ${ }^{3}$ Such systems can reduce in-hospital mortality and serious adverse events. ${ }^{4}$ The Royal College of Physicians recommend EWS for monitoring of all adult patients, and this has led to the development of a standardised National EWS in the UK. ${ }^{5}$

Standard EWS are not recommended for use in women who are pregnant as physiological parameters and their response to illness are modified in pregnancy. ${ }^{5}$ Previous reports from the UK Confidential Enquiries into Maternal Deaths in the UK recommend using a modified EWS to assist in the identification of obstetric patients at risk of deterioration. ${ }^{6}$

A postal survey in the UK found that only $19 \%$ of maternity units were using an EWS in obstetric patients, and only $6 \%$ of units were using a system modified for parturients. ${ }^{7}$ The scoring systems used were not consistent in terms of physiological variables used, the layout of the charts or the trigger thresholds. At the time of writing, there is no nationally validated EWS for pregnant patients in the UK. However, many example charts have been proposed.

In 2013, one group of researchers from the UK used logistic regression to predict mortality in a sample of pregnant patients from a critical care database of over 4000 intensive care patients. In their analysis of the relationship between changes in individual vital signs and mortality, they were able to produce an early warning scoring system that is statistically weighted according to variables reflective of vital signs. This was then internally validated to produce a clinical EWS system that is tailored to obstetric patients. Work is ongoing to validate its use in other obstetric units. ${ }^{8}$

Work on EWS has taken place in many destinations outside the UK. A hospital in Auckland, New Zealand, has adopted a maternal early warning system (MEWS) into standard practice after a review of obstetric cases referred to intensive care found that a MEWS could have reduced the seriousness of maternal morbidity in $7.6 \%$ of cases. ${ }^{9}$

Currently, there is only a small amount of research into the benefits of EWS in low-resource settings. Kruisselbrink et $a l^{10}$ carried out a pilot study using a modified EWS at Mulago Hospital, Uganda. They evaluated a modified EWS as a mortality predictor and concluded the EWS could identify high-risk patients in a busy critical care environment. The use of Triage Early Warning Score (TEWS) has been suggested as one component of a scale called the South African Triage Scale produced by the South African Triage Group from the Division of Emergency Medicine at the Universities of Cape Town and Stellenbosch and recommended by the Emergency Medicine Society of South Africa. ${ }^{11}$ This system assists with determining the urgency of medical treatment during triage in emergency departments. It has also been proposed as a useful assessment tool to analyse the efficacy of emergency care in developing countries. ${ }^{12}$

A report by Esegbona ${ }^{13}$ describes development of a training package that includes the introduction of a form of EWS, a track and trigger system called ChEWA (chatinkha early warning alarm). An alternative system has been trialled in Mbarara University Hospital, Uganda. ${ }^{14}$ The majority of patient care was identified as being provided by patient attendants and relatives, thus they deduced to implement a 'lay MEWS' for these attendants to compensate for nursing staff shortages.

The current study describes the process and potential impact of introducing a modified obstetric EWS (MOEWS) at FHRH, an Ethiopian referral hospital.

\section{Measurement}

A three-phase retrospective case note review was conducted over a 1-year period (December 2014December 2015) coupled with staff questionnaires and semistructured interviews.

Prior to introduction of the MOEWS, a case note review of 20 postoperative obstetric cases was conducted over a 3-day period in December 2014 (16 LUSCS (lower uterine segment caesarean section), 2 MROP (manual removal of placenta) and 2 laparotomy). Vital signs were recorded regularly ( $15 \mathrm{~min}$ for 2 hours then hourly for 4 hours) for most cases in recovery (90\%). However, this was usually incomplete with all of heart rate (HR), blood pressure $(\mathrm{BP})$, respiratory rate (RR) and peripheral oxygen saturation $\left(\mathrm{SpO}_{2}\right)$ being recorded in only $55 \%$ cases. Oxygen saturations were recorded in only $35 \%$ cases and temperature in only $25 \%$. Urine output was not recorded hourly in any cases. Recognition that suboptimal monitoring occurred in the postoperative period formed the rationale for introduction of the MOEWS system in FHRH.

The MOEWS system introduced was a recording chart for the patient's vital signs. Within the chart were coloured 'zones'. If vital signs were recorded within one of these coloured zones (a 'trigger), then an action should be prompted. Yellow zone 'triggers' should prompt an increase in the frequency of monitoring, and red zone 'triggers' should prompt a medical review by a doctor. By transferring recorded vital signs from the case notes reviewed above to a sample MOEWS, it was noted that $50 \%(n=10)$ of cases would have triggered a red warning $(\mathrm{n}=10)$ in recovery, and $36 \%$ of these were recorded within $15 \mathrm{~min}$ of transfer from theatre. Medical review occurred in $30 \%(n=3)$. In $70 \%(n=7)$ of cases with a red zone trigger vital sign, no action was taken. No cases triggered a yellow warning.

Follow-up case note review was conducted at 8 months and 11 months postintroduction of MOEWS (20 cases and 40 cases, respectively) to determine practices and the potential impact of the MOEWS.

Paper-based questionnaires containing both closed and free-text response questions were distributed among healthcare workers (midwives, interns and residents) in the facility and were completed anonymously and at 
participant convenience. Questionnaires were conducted at $8(n=24)$ and 11 months $(n=34)$ to establish opinion on the system and any change in this over time. Consent was implied by questionnaire completion.

Semistructured face-to-face interviews $(n=7)$ were conducted at 11 months (November 2015) with those who consented and were tape-recorded. Information leaflets were provided, and written consent was obtained. Interview questions were predesigned based on themes reflected in survey responses.

Data from the case note reviews were analysed using Microsoft Excel software. Responses to survey questions were analysed using descriptive statistics. Subgroup analysis was carried out between occupational groups. Free-choice responses and interview data were analysed qualitatively by identification of key themes.

\section{Design}

Following initial review, a MOEWS was designed after joint discussion with obstetricians, anaesthetists and management from FHRH. This included developing a MOEWS to meet the needs and resources at FHRH. The primary aim was to establish standardised observation and management of obstetric patients postoperatively, and a guideline was produced to accompany the MOEWS. The MOEWS was intended to be used in all postoperative patients on the obstetric ward, thus included all operative deliveries and postdelivery complications. The MOEWS was standardised and not adapted for specific conditions or surgeries. The main adaptations included adjustments to scales for vital signs recording to allow for the extremes in vital signs, which can be witnessed with late presentations to the referral hospital.

The MOEWS served to detect potential postoperative complications and prompt action earlier. The standard set for monitoring was every $15 \mathrm{~min}$ for 2 hours, then hourly for 4 hours thereafter and included appearance, respiratory rate, heart rate, blood pressure, conscious level, temperature, saturations, pain score and urine output. A vital sign in the red coloured zone was intended to trigger medical review, whereas the yellow area would indicate a need for more frequent monitoring, for example, return to $15 \mathrm{~min}$ intervals, or increase to $5 \mathrm{~min}$ intervals if within first 2 hours. In addition, if a trend was noted in the observations that indicated deterioration, medical review was indicated.

Training was provided to accompany the introduction of the MOEWS and its associated guideline, provided by both internal senior clinicians and external visiting clinicians familiar with MOEWS, and this was repeated following each monitoring period.

\section{Strategy}

Repeated periods of monitoring and evaluation allowed assessment of the impact of the MOEWS on patient care, and allowed identification of problems and barriers to use of the MOEWS or compliance with monitoring guidelines. Through staff feedback in the form of questionnaire survey and interviews, attitudes towards the system could be ascertained, and staff were able to make suggestions for improvement, for example, alteration to MOEWS chart layout.

\section{RESULTS}

\section{Effect on monitoring and patient care}

Following introduction of the MOEWS, a review of 20 cases was conducted at 8 months. Utilisation of the MOEWS was $100 \%$. Monitoring and recording of vital signs was greatly improved, with most parameters recorded regularly as per guideline. In particular, oxygen saturations recording had risen from $35 \%$ to $95 \%$, temperature from $25 \%$ to $85 \%$ and urine output from $0 \%$ to $30 \%$. Sixty-five per cent $(n=13)$ cases had vital signs in the yellow section and therefore required closer monitoring. This occurred in $69 \%(n=9)$. A further $15 \%(n=3)$ showed vital signs in the red section and therefore required medical review. Medical review occurred in $67 \%(n=2)$. The final case was reviewed later at the scheduled ward round. Overall, $69 \%$ $(11 / 16)$ cases with a trigger were acted on.

At 11-month postintroduction of MOEWS, a further review was undertaken to determine if practices were maintained. This included 40 cases (36 LUSCS, 2 hysterectomy and 2 laparotomy). Of these, 28\% ( $n=11)$ had a complication (three uterine rupture, three pre-eclampsia, 1 PROM, 3 APH, 1 APH +PROM). MOEWS was utilised in $100 \%$ cases. Standards of monitoring were maintained with the exception of urine output (figure 1).

A total of $22.5 \%$ recorded cases $(n=9)$ 'triggered' in recovery, $15 \%$ of total cases $(n=6)$ were red triggers, $33 \%(\mathrm{n}=2)$ of these had evidence of management. A percentage of 7.5 of total cases $(n=3)$ were yellow triggers. There was evidence of action in response to the vital sign trigger in $55.6 \%$ ( $\mathrm{n}=5)$ of trigger cases, for example, increased monitoring frequency or medical review. Only $22 \%(n=2)$ had evidence of a specific medical intervention, for example, fluid or medication prescription or entry in medical notes. All patients who had 'triggered' in recovery were eventually discharged home.

The number of patients who alerted a red trigger decreased from $50 \%$ pre-MOEWS to $15 \%$ at 8 and 11 months; however, the proportion of these who received prompted action was variable. It is difficult to interpret this as review may have occurred, but no action documented or deemed necessary. It is unclear whether the fall in number of patients triggering is a reflection of improved care.

\section{Staff views on the MOEWS}

At 8 months, 24 questionnaires were completed $(83 \%$ $[\mathrm{n}=20]$ midwives, $17 \%[\mathrm{n}=4]$ interns) then at 11 months, 34 completed responses were received $(42 \% \quad[n=14]$ midwives, $30 \%[\mathrm{n}=10]$ interns, $15 \%[\mathrm{n}=5]$ residents, $12 \%$ [n=4] unknown, 3\% medical student $[\mathrm{n}=1]$ ), a response rate of $85 \%$, from the 40 questionnaires that were distributed to staff in November 2015. 


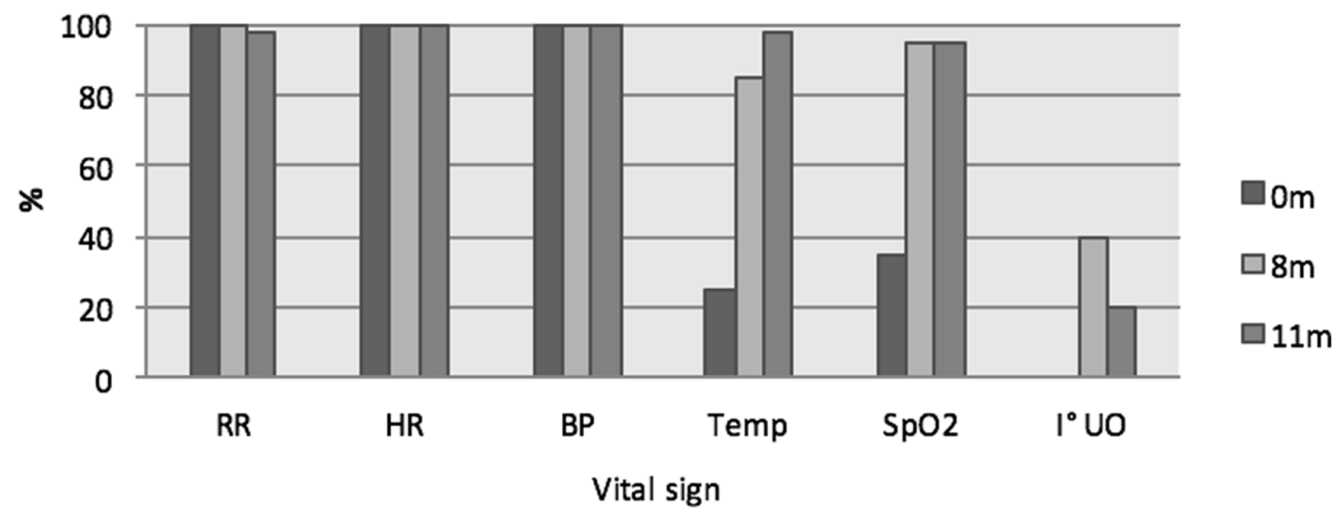

Figure 1 Completion of vital signs pre-MOEWS $(0 \mathrm{~m})$ and at $8(8 \mathrm{~m})$ and 11 months $(11 \mathrm{~m})$ post-MOEWS implementation. MOEWS, modified obstetric early warning system.

Between the surveys, the number of staff responding 'yes, often' or 'yes, sometimes' to using the MOEWS rose from $57 \%$ to $82 \%$ and $15 \%$ to $35 \%$, respectively. The proportion who reported having been trained rose from $71 \%$ to $79 \%$ and the proportion confident using it rose from $58 \%$ to $85 \%$, with $94 \%$ feeling they could show others how to use it compared with $73 \%$ in the earlier study.

The proportion of staff who felt the system was either 'very useful' or 'useful' rose from $91 \%$ to $97 \%$, and the number reporting problems using it dropped $(46 \%$ to $21 \%)$. However, the number who would recommend it dropped slightly ( $82 \%$ to $77 \%$ ). This did not appear to be related to views of any increased workload created by the system as $69 \%$ increasing to $91 \%$ felt it reduced workload.

Free-text responses to advantages included the benefits of the MOEWS for early detection of problems $(n=25)$, its ease of use $(n=12)$, its ability to prompt staff $(n=3)$ and assisting with the management of a patient $(n=13)$. Disadvantages that were mentioned included increased workload for staff $(n=2)$, that currently there is no exact value for the vital sign written on the MOEWS $(n=6)$ and that it may be difficult to understand, especially if not trained $(\mathrm{n}=5)$. Other individual points made related to potential changes to improve the form layout, for example, 'more space' and 'action column'. Subgroup analysis was performed, and no notable differences were found between occupational groups.

Seven interviews were conducted following the final monitoring phase (four midwives, three interns). Responses were overall positive (box 1).

\section{Lessons and limitations}

The MOEWS has resulted in a considerable improvement in the monitoring of patients' vital signs. The overall number of patients 'triggering' dropped significantly following introduction of the MOEWS that could suggest improved monitoring and management preventing patients reaching the point at which they trigger, although this cannot be confirmed. Action prompted by triggers was variable and difficult to interpret. The MOEWS helped to identify further areas in need of training, for example, pain assessment.

Attitudes towards the MOEWS were positive in the majority of staff in the department with a feeling it helped staff to cope with the demands of their work while making it easier to detect unwell patients. In the majority of themes explored, attitudes were seen to be improving over time.

Identified limiting factors in the recording of vital signs was the availability of functioning equipment, lack of knowledge of specific scales used (eg, numerical pain scale and conscious level), time constraints and complexity of documentation for varying conditions within FHRH, sometimes resulting in repetition. Shift patterns vary considerably, staff turnover can be high and regular training is necessary to ensure all staff are familiar with the MOEWS.

Using retrospective case note review meant that assessment of management was complex, and documentation, or lack of, may not reflect actual clinical practice.

Survey responses may have been biased by desire to 'please' the researcher or concerns of handwriting recognition resulting in censoring. Despite the practice of

\section{Box 1 Staff comments on the modified obstetric early} warning system (MOEWS)

- 'It is so fantastic. It is easy to understand, after we have filled vital sign, the operator can easy understand and compare the trends' (11).

- 'It is very important for the patient and for us, as you know we have limited manpower and, [with] the number of patients that flow in $\mathrm{FHRH}$, it simplifies our workload ...we can understand problems early and we can put our solution for [the] problem' (I2).

- 'When comparing with the other vital sign sheets that we are using in the past [and] still which we are using... in paediatrics and medicine, the MOEWS is better than that. Despite its disadvantages, it has more advantages than the previous vital sign sheet' (I3).

- '[It is] easy to detect any danger sign because simply detect by colour' (M2).

- 'Early warning systems could be used in other departments if possible. It is very important. We need it' (I2). 
medicine in English language, responses to survey and interviews could have been limited by respondent's ability to convey themselves in a second language. Interviews that took place in the workplace may have been affected by ongoing clinical duties or time constraints leading to poor focus.

\section{CONCLUSIONS}

Reducing maternal mortality is a significant challenge in Ethiopia. One basic way to prevent maternal deaths in healthcare facilities is to detect physiological parameters suggestive of deterioration in condition before such changes become irreversible and fatal. This is the aim of the MOEWS system. The MOEWS system can prompt increases in monitoring frequency and medical review based on the colour-coded trigger system and could also be used to evaluate quality of care through assessing whether triggers result in actions and interventions. Surveillance of cases which trigger could lead to further evaluation and discussion of quality of care for example, at maternal morbidity and mortality meetings.

It is encouraging that FHRH has embraced the MOEWS, and a positive effect appears to have occurred on patient safety in the recovery area with improved vital sign recording following its introduction. Staff opinions on the MOEWS are seen to be positive in the majority, showing that it is an acceptable tool to cope with the unique demands faced at FHRH, with high patient flow and low resources. As there is little literature considering the impact of EWS in low-resource settings, it is hoped that the findings from this research will lead to further development, implementation and evaluation in this area.

Acknowledgements The authors wish to acknowledge Johnson \& Johnson and THET for funding this project and the staff and management of Felege Hiwot Referral Hospital for their support throughout the project.

Contributors JM was responsible for designing and managing the project, preintervention data collection, MOEWS implementation and training, design of questionnaires and interview questions and overall analysis. IP undertook data collection and analysis at 8 months post-MOEWS implementation, assisted with questionnaire design and conducted questionnaires. DT undertook data collection and analysis at 11 months post-MOEWS implementation and conducted questionnaires and interviews. BF was local lead clinician and was involved in modification of the MOEWS, its implementation and training. WG supervised the project, assisted with the study design and analysis interpretation.

Funding This project was supported by THET Strengthening Surgical Capacity grant SSC 4.1 funded by Johnson \& Johnson.
Competing interests None declared.

Patient consent for publication Not required.

Ethics approval Ethical approval was granted for this study by the University of Aberdeen College of Life Sciences and Medicine Ethics Review Board and permission granted from the Felege Hiwot Referral Hospital CEO and Head of Obstetrics.

Provenance and peer review Not commissioned; externally peer reviewed.

Open access This is an open access article distributed in accordance with the Creative Commons Attribution Non Commercial (CC BY-NC 4.0) license, which permits others to distribute, remix, adapt, build upon this work non-commercially, and license their derivative works on different terms, provided the original work is properly cited, appropriate credit is given, any changes made indicated, and the use is non-commercial. See: http://creativecommons.org/licenses/by-nc/4.0/.

\section{REFERENCES}

1. WHO Country Profile. Ethiopia, 2015. www.who.int/countries/eth/en

2. Central Statistical Agency (CSA) [Ethiopia] and ICF. Ethiopia Demographic and Health Survey 2016: Key Indicators Report. Addis Ababa, Ethiopia, and Rockville, Maryland, USA: CSA and ICF, 2016.

3. Alam N, Hobbelink EL, van Tienhoven AJ, et al. The impact of the use of the Early Warning Score (EWS) on patient outcomes: a systematic review. Resuscitation 2014;85:587-94.

4. Petersen JA, Mackel R, Antonsen K, et al. Serious adverse events in a hospital using early warning score - what went wrong? Resuscitation 2014;85:1699-703.

5. Royal College of Physicians. National Early Warning Score (NEWS): Standardising the assessment of acute illness severity in the NHS. Report of a working party. London: RCP, 2012.

6. Lewis G, (ed). The Confidential Enquiry into Maternal and Child Health (CEMACH). Saving Mothers' Lives: reviewing maternal deaths to make motherhood safer - 2003-2005. The Seventh Report on Confidential Enquiries into Maternal Deaths in the United Kingdom. London: CEMACH, 2007.

7. Swanton RD, Al-Rawi S, Wee MY. A national survey of obstetric early warning systems in the United Kingdom. Int J Obstet Anesth 2009;18:253-7.

8. Carle C, Alexander P, Columb M, et al. Design and internal validation of an obstetric early warning score: secondary analysis of the Intensive Care National Audit and Research Centre Case Mix Programme database. Anaesthesia 2013;68:354-67.

9. Austin DM, Sadler L, McLintock C, et al. Early detection of severe maternal morbidity: a retrospective assessment of the role of an Early Warning Score System. Aust N Z J Obstet Gynaecol 2014;54:152-5.

10. Kruisselbrink RJ, Kwizera A, Crowther M, et al. Monitoring of medical and surgical patients in Mulago hospital, Uganda: A pilot observational study using the modified early warning score. Am J Respir Crit Care Med 2014;189:A3777.

11. The Emergency Medicine Society of South Africa. The South African Triage Scale (SATS). 2012. Available: http://emssa.org.za/sats/

12. Sun JH, Twomey M, Tran J, et al. The need for a usable assessment tool to analyse the efficacy of emergency care systems in developing countries: proposal to use the TEWS methodology. Emerg Med $J$ 2012;29:882-6.

13. Esegbona G. Critical help early for women in Africa-the Chewa system. The need for critical care in Africa. Int J Gynecol Obstet 2015;131:E348.

14. Walker I, Ross N, Kiwanuka J, et al. Development of a 'lay mews' for patient attendants in the obstetric wards in Mbarara Regional Referral Hospital Uganda. Br J Anaesth 2012;108:209. 